\title{
Tratamiento definitivo en traumatismo avulsivo de la región cérvico-facial. Presentación de caso clínico
}

\author{
Amilkar Suárez Pupo ${ }^{1,2}$, Carlos Antonio Arias $\mathrm{Pla}^{1}$, \\ Marcelo Jorge Vargas Baños ${ }^{1}$ y Karol Magdalena Yagual Jiménez ${ }^{1}$
}

'Hospital del Instituto Ecuatoriano de Seguridad Social Babahoyo

ZUniversidad Técnica de Babahoyo. Babahoyo, Ecuador.

Recibido 2021-03-09 aceptado 2021-04-19

Correspondencia a: Dr. Amilkar Suárez Pupo asuarez@utb.edu.ec

\section{Definitive treatment in avulsive trauma of the cervico-facial region. Clinical case report}

Introduction: Self-inflicted injuries by long-barreled firearms in the cervico-facial area do not always succeed in consummating suicide and may result in an avulsive injury of this anatomical region. Aim: Socializing the benefits of the immediate multidisciplinary treatment in the medical care of patients with complex facial trauma. Clinical case: A 60 -year-old male patient with an autolytic attempt by firearm, with loss of tissue at the mandibular level, as well as airway compromise. Results: The different stages of the surgical action were achieved during the first four hours since the incident occurred. The patient was discharged without the need for a new surgical procedure. Discussion: Currently there is e tendency to perform definitive treatment in a single initial surgical procedure. Conclusion: The immediate and protocolized intervention of specialties such as General Surgery, Maxillofacial and Plastic-Reconstructive Surgery in patients with avulsive wounds of the cervical-facial region can achieve a definitive treatment in a single surgical time and with favorable results.

Keywords: gunshot wound; lower jaw; tracheostomy; definitive treatment.

\section{Resumen}

Introducción: Las lesiones autoinfligidas por armas de fuego con cañón largo en la zona cérvico-facial no siempre logran consumar el suicidio y pueden ocasionar una herida avulsiva de esta región anatómica. Objetivo: Socializar los beneficios del tratamiento multidisciplinario inmediato en la atención al paciente con traumatismo facial complejo. Caso clínico: Paciente masculino de 60 años de edad con intento autolítico por arma de fuego, con pérdida importante de tejido a nivel mandibular y compromiso de la vía aérea. Resultados: Las distintas etapas de accionar quirúrgico se lograron en las primeras cuatro horas desde que sucedió el incidente. El paciente no presentó complicaciones posoperatorias ni necesidad de una nueva operación. Discusión: En la actualidad existe la tendencia a realizar tratamiento definitivo en un solo tiempo quirúrgico inicial. Conclusión: La intervención inmediata y protocolizada de las especialidades cirugía general, maxilofacial y cirugía plástica-reconstructiva en pacientes con heridas avulsivas de la región cérvico facial pueden lograr un tratamiento definitivo en un único tiempo quirúrgico y con resultados favorables.

Palabras clave: herida por arma de fuego; maxilar inferior; traqueostomía; tratamiento definitivo.

\section{Introducción}

El intento autolítico y el suicidio son considerados un problema de salud pública y mental de impacto en la sociedad global contemporánea ${ }^{1}$. Variedades de métodos se emplean para lograr estos fines. Los más violentos son característicos de los suicidios consumados, siendo la población masculina la que con mayor frecuencia los emplea. Entre los más usados tenemos: la precipitación, el ahorcamiento y el uso de armas de fuego ${ }^{2}$. En las mujeres, las lesiones auto infligidas por armas de fuego son más frecuentes en el abdomen, mientras que la localización del disparo en la región cérvicofacial tiene mayor incidencia en los hombres ${ }^{3}$.

Las lesiones balísticas varían dependiendo de la 
velocidad de traslación del proyectil, según la distancia a la que fue realizado el disparo. Se considera como de alta velocidad de traslación los que superan los 1.200 pies por segundo ${ }^{4}$. El grado de severidad de las lesiones aumenta si la distancia en que fue realizado el disparo al lesionar a la víctima es menor a 2,74 metros 5 . Al contacto con el hueso, puede provocar que fragmentos óseos se desplacen de forma errática. Además, el proyectil se puede deformar y fragmentar causando mayor daño al tejido blando ${ }^{6}$.

Se reconocen como armas largas las que tienen un cañón con una longitud mayor a $30 \mathrm{~cm}^{7}$. Paradójicamente, la utilización de estas se relaciona con una menor mortalidad que la de las armas cortas y esto se debe a la distancia que existe entre el final del cañón y el gatillo, lo que dificulta el accionar para la víctima. A lo anterior se le suma la hiperextensión cervical refleja, que modifica la trayectoria esperada del proyectil. El grado de angulación de la cabeza es un factor determinante para lograr el fallecimiento inmediato o provocarse lesiones graves a nivel facial ${ }^{3}$.

La atención inicial a pacientes con heridas avulsivas en la región cérvico-facial puede ser un desafío para el personal sanitario a cargo debido a la complejidad de este tipo de lesiones. La aplicación organizada de los protocolos de apoyo vital avanzado permitirá la apertura a una serie de acciones que intenten garantizar tanto la vida del paciente traumatizado como la restauración de la continuidad, función y estética facial.

\section{Reporte de caso}

Paciente masculino de 60 años de edad que arribó a emergencias con herida avulsiva en la región cérvico-facial por intento autolítico con arma de fuego, con dificultad para respirar que lo obligaba a mantener la posición de sentado debido a la hemorragia existente a nivel mandibular.

Al incrementarse el compromiso de la vía aérea se inició la secuencia rápida de intubación orotraqueal asistida por medicamentos. La inestabilidad del área dificultó el sello de la máscara facial con reservorio. Se realizaron dos intentos de intubación orotraqueal (Figura 1), los cuales fueron fallidos. Se colocó una máscara laríngea de forma transitoria y se garantizó la vía aérea con la técnica quirúrgica de cricotiroidostomía.

El control de la hemorragia se logró con ligadura y compresión directa. Se descartó la existencia de otras lesiones y se completó en emergencias la reanimación, administración de medicamentos y los estudios de imagen (Figura 2).
De inmediato, el paciente fue trasladado al quirófano central. Los procedimientos realizados fueron. a. Desbridamiento quirúrgico, más retiro de fragmentos óseos y de proyectiles

b. Regularización de bloque óseo mandibular

c. Reducción y estabilización con sistema de mini placas de titanio 1,5, 2,0 y tornillos mono y bicortical

d. Rehabilitación del cuerpo de la mandíbula en su porción medial y el mentón con malla de titanio (Figura 3).

e. Glosorrafia.

f. Vestibuloplastía.

g. Queiloplastía.

h. Colgajos cutáneos de avance y Z-plastía.

i. Cambio de la cricotiroidotomía por traqueostomía.

El paciente recibió un tratamiento inmediato, protocolizado y multidisciplinario que le garantizó una reconstrucción completa en un periodo menor a $4 \mathrm{~h}$ desde el momento en que ocurrió el trauma (Figura 4). La recuperación posoperatoria inicial fue

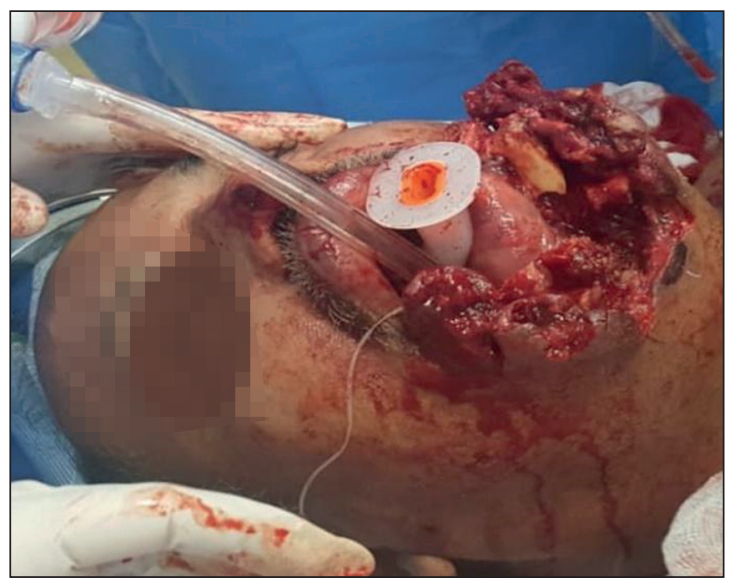

Figura 1. Herida avulsiva de la región cérvico facial que dificulta las maniobras para garantizar una vía aérea definitiva.

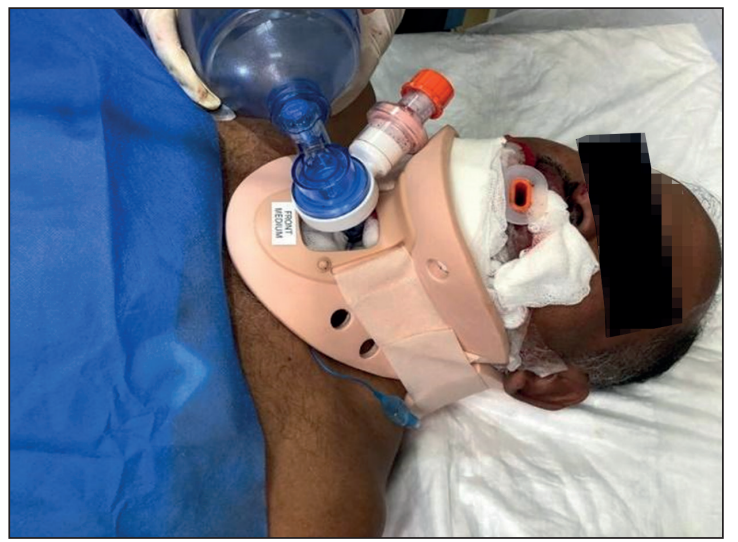

Figura 2. Vía aérea definitiva por técnica quirúrgica de cricotiroidostomía, control de la movilidad de la columna cervical y de la hemorragia. 


\section{CASOS CLÍNICOS}

Figura 3. Reducción, estabilización y rehabilitación del cuerpo de la mandíbula.

Figura 4. Tratamiento reconstructivo con técnica de colgajos cutáneos de avance y Z-plastia.
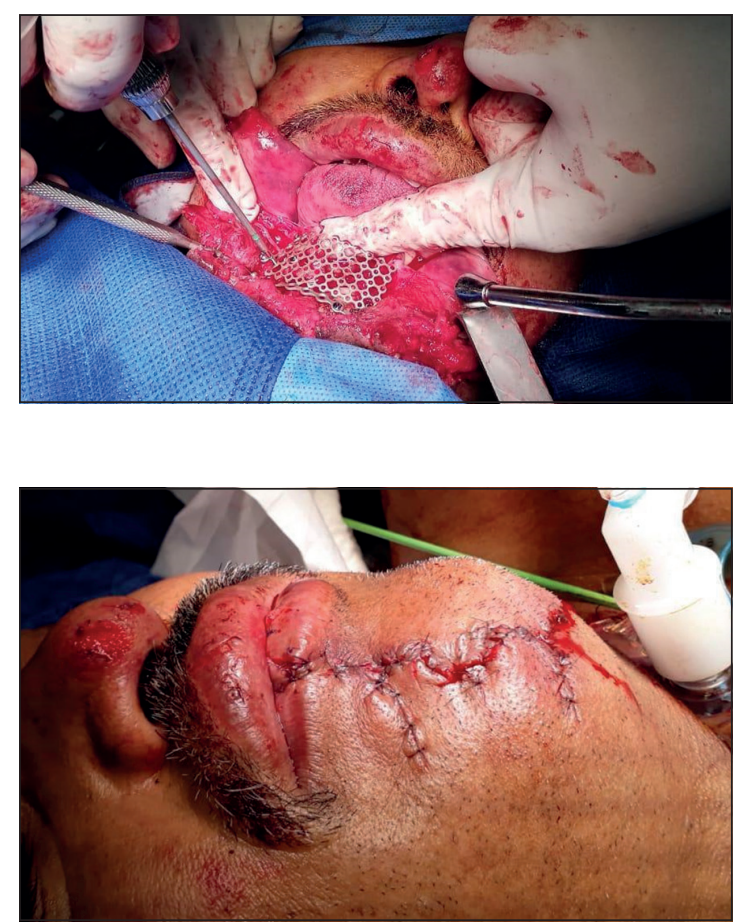

en unidad de cuidados intensivos; luego se trasladó a sala de hospitalización donde tuvo una evolución favorable; se logró decanular sin complicaciones y fue dado de alta. En el seguimiento por consulta, se pudo comprobar que la competencia labial, el habla, la alimentación y la estética tenían resultados óptimos. No fueron necesarias nuevas intervenciones quirúrgicas.

\section{Discusión}

El tratamiento de la vía aérea e inmovilización de la columna cervical en pacientes con lesiones complejas cérvico-faciales dependerá en cierta medida de la colaboración del afectado. Un paciente que responde con adecuada fonación o con señales corporales, permite descartar obstrucción de la vía aérea, trastornos ventilatorios, así como alteración hemodinámica y del estado de conciencia. En estos casos, la aplicación de la Regla Canadiense de la Columna Cervical permite decidir si el paciente necesita del uso del collarín cervical ${ }^{8}$.

La vía aérea definitiva (VAD) será el objetivo inicial a lograr en este grupo de pacientes. Autores como Mardones- $\mathrm{M}^{9}$ y Rosario Soto ${ }^{10}$ plantean que la intubación orotraqueal es un método que se puede llevar a cabo en la mayoría de los casos en primer orden.
Planteamos como autores de este trabajo que las pérdidas del soporte estructural de la región maxilofacial por heridas avulsivas, conlleva a que las técnicas usuales de mantenimiento de la vía aérea como cánulas orofaríngeas y máscaras con reservorio no sean efectivas debido a la fuga que provocan. Por tal motivo, sugerimos el uso de dispositivos supraglóticos que garanticen una permeabilidad adecuada de la vía aérea. Algunos de estos permiten el paso del tubo endotraqueal los cuales facilitarán una $\mathrm{VAD}^{11}$.

Pueden ser varios los criterios para la realización de una vía aérea quirúrgica ${ }^{10,11}$. Se debe iniciar con la oxigenación transtraqueal percutánea con aguja $\mathrm{y}$, a continuación, la cricotiroidostomía quirúrgica de emergencia. La traqueostomía de emergencia se debe realizar en el quirófano con una vía aérea definitiva garantizada o, de forma electiva, para reemplazar a una cricotiroidostomía ya existente.

La hemorragia masiva exanguinante debe ser de atención prioritaria ${ }^{7,12}$. Los métodos a emplear serían: la realización de una arteriografía diagnóstica con la embolización selectiva del vaso lesionado o, en su defecto, la exploración quirúrgica urgente y hemostasia del vaso ${ }^{13,14}$. Los autores de este trabajo concordamos con lo que plantea García-Núñez ${ }^{14}$ en cuanto al manejo de la hemorragia de moderada o poca cuantía. El sangrado se puede controlar a través de compresión directa, taponamientos nasales anteriores y posteriores, taponamientos orofaríngeos, empaquetamiento, electrocoagulación, sutura o ligadura directa, adyuvantes biológicos y químicos.

En el tratamiento quirúrgico emergente se realizará desbridamiento, retiro de dientes no viables, hueso desvitalizado, lavado de herida; así como retiro de material contaminante, material necrótico y cuerpos extraños. Se efectuará la inmovilización de los fragmentos óseos ${ }^{15}$. Las lesiones faciales más complejas, con pérdidas de sustancia y fracturas graves asociadas con control de la hemorragia, en ocasiones, requieren una estabilización hemodinámica antes de realizar el tratamiento quirúrgico. Estas pueden esperar un máximo de $24 \mathrm{~h}$ para ser suturadas sin compromiso del resultado final ${ }^{4}$.

El tratamiento inmediato de todas las lesiones mejora los resultados estéticos y funcionales. En la actualidad existe la tendencia a realizar tratamiento definitivo en un solo tiempo quirúrgico inicial, a pesar de la complejidad del traumatismo, en el que se incluye la toma de injertos libres. El tratamiento secundario se debe efectuar en casos complejos a reconstruir mediante injertos óseos, rotación de colgajos miocutáneos o colgajos microvascularizados ${ }^{6}$. 


\section{Conclusiones}

Las condiciones del paciente, las características de la lesión, los recursos disponibles y la experiencia del personal a cargo condicionan el éxito en el tratamiento definitivo de las heridas avulsivas de la región cérvico-facial. Este trabajo da fe de la pertinencia de una intervención inmediata, protocolizada y multidisciplinaria en pacientes con traumatismos complejos a este nivel, para lograr un tratamiento definitivo en un único tiempo quirúrgico con resultados favorables

\section{Responsabilidades éticas}

Protección de personas y animales. Los autores declaran que para esta investigación no se han realizado experimentos en seres humanos ni en animales.

Confidencialidad de los datos. Los autores declaran que en este artículo no aparecen datos de pacientes.

Conflictos de interés: no hay.

El artículo está aprobado por el Comité de Ética Institucional.

\section{Bibliografía}

1. Molina Linares II, Mora Marcial G, Carvajal Herrera A, Marrero Salazar M, García Triana G. Factores de riesgo asociados a la conducta suicida en el adulto mayor. Medicentro Electrónica. 2020;24:54-67.

2. Guamán Ledesma JL, Vázconez Pino GP, Cando Pilatuña RI. Intento autolítico en pacientes atendidos en el Servicio de Psiquiatría. Hospital General Docente Riobamba, 2018 [Internet]. Vol. Tesis Psic, Repositorio Digital UNACH. Universidad Nacional de Chimborazo; 2019. Available from: http://dspace.unach. edu.ec/handle/51000/5335

3. Villanueva-Alcojol L, Zaldivar DMS De, González-García R, Ballester DG, Correa IR, Vila CH, et al. Combinación de colgajos locales y libre microvascularizado para reconstrucción del tercio facial inferior tras traumatismo por arma de fuego. Rev Española Cir Oral y Maxilofac. [Internet]. 2011;33:162-

7. Available from: http://dx.doi. org/10.1016/j.maxilo.2011.05.003

4. Vatsyayan A, Adhyapok AK, Debnath SC, Malik K. Reconstruction and rehabilitation of short-range gunshot injury to lower part of face: A systematic approach of three cases. Chinese J Traumatol. - English Ed [Internet]. 2016;19:239-43. Available from: http:// dx.doi.org/10.1016/j.cjtee.2016.01.016.

5. Escobar Vega H, Duarte N, Expósito Jalturin A, Martínez León R, Abela Lazo AM. Herida por arma de fuego. Reconstrucción quirúrgica
Presentación de un caso clínico. Rev Electrónica Portales Médicos [Internet]. 2010; Available from: https://www. portalesmedicos.com/publicaciones/ articles/1972/4/Herida-por-arma-de-fuego Reconstruccion-quirurgica.-Presentacionde-un-caso-clinico.

6. Palacios Vivar DE, Miranda Villasana JE, Calderón Lumbreras AS. Herida facial por proyectil de arma de fuego: revisión de literatura y estudio clínico de tres casos. Rev Odontológica Mex. [Internet]. 2017;21:127-34. Available from: https:// www.portalesmedicos.com/publicaciones/ articles/1972/4/Herida-por-arma-defuego.-Reconstruccion-quirurgica.Presentacion-de-un-caso-clinico

7. Brofman C, Chiari R, Ferrante M, Fernández J, Chiari R, Algieri A. Bases Anatómicas para el Control Vascular de la Cara en el Trauma. J Heal Med Sci. 2019;5:133-40.

8. American College of Surgeons A. Trauma de la columna vertebral y médula espinal. In: Soporte Vital Avanzado en Trauma (ATLS). DÉCIMA EDI. Chicago; 2019. p. 129-46.

9. Mardones MM, Bravo AR, Fernández TM, Gunckel MR, Torres MC. Sistemática en el Tratamiento Quirúrgico de las Fracturas Panfaciales Aplicado a un Caso Clínico De Alta Complejidad. Int J Odontostomatol. 2020;14:590-5.

10. Rosario Soto AS. Prevalencia de fracturas en maxilar inferior por heridas de arma de fuego en rostro, en pacientes atendidos en el Hospital General de accidentes "ceibal [Internet]. Vol. 8, Maestría thesis. Universidad de San Carlos de Guatemala.;
2019. Available from: http://www. repositorio.usac.edu.gt/12365/

11. American College of Surgeons A. Manejo de la vía aérea y la ventilación. In: ATLS, Soporte Vital Avanzado en Trauma 10ma Edición. Décima. Chicago; 2018. p. 2341.

12. Burgess CA, Dale OT, Almeyda R, Corbridge R. An evidence based review of the assessment and management of penetrating neck trauma. Clin Otolaryngol. [Internet]. 2012;37:245. Available from: https://onlinelibrary. wiley.com/doi/full/10.1111/j.17494486.2011.02422.x

13. Ruiz Laza L, Herrera Cobos J, Díaz Fernández JM, González Padilla JD, Belmonte Caro R, García-Perla García A, et al. Manejo terapéutico inicial de las heridas por arma de fuego en el territorio maxilofacial. Rev Esp Cir Oral y Maxilofac. 2006;28:27786.

14. Garcia-Núñez LM, Sánchez-Villanueva GE, Cabello-Pasini R, Soto-Ortega LE, Rivera-Cruz JM, Núñez-Cantú O. Angioembolización selectiva: Un valioso adyuvante en la estrategia de control de daños en heridas faciales por proyectil de arma de fuego. Informe de un caso. Cir Cir. 2009;77:217-21

15. Quintana Díaz JC, Villareal Corbo N, Quintana Giralt M. Fractura mandibular por herida de arma de Fuego: Presentación de un caso. Acta Odontol Colomb. [Internet]. 2019;3:05. Available from: http://scielo.isciii. es/scielo.php?script=sci arttext\&pid $=\mathrm{S} 1130-05582008000600006$. 